\title{
EFFECT OF BORON ON THE ELECTRONIC PROPERTIES OF POLYCRYSTALLINE GRAPHITE*
}

\author{
P. S. Grosewald ${ }^{+}$and P. L. Walker, Jr. \\ The Pennsylvania State University \\ University Park, Pennsylvania 16802, U.S.A.
}

(Received April 5, 1970)

\begin{abstract}
In a polycrystalline material such as the compacted natural graphite flake used in this study, the electronic transport properties of greatest interest are the absolute thermoelectric power (since it is relatively independent of crystalline size and shows the effect of the individual Fermi surfaces), its temperature dependency and the Hall constant. Studies on the undoped compacts in the temperature range $77-550 \mathrm{O}$ show that the absolute thermoelectric power decreases with increasing temperature, passing from positive to negative values at about $250^{\circ} \mathrm{K}$. The room temperature value $\left(\alpha_{300}\right)$ is $-1.5 \mu \mathrm{V} /{ }^{\circ} \mathrm{K}$.

In our work, boron powder was mixed with SP-1 graphite, compacted and annealed to give final B/C atom ratios of $-10^{-6}, 10^{-5}$, and $5 \times 10^{-4}, 1,2$ and $5 \times 10^{-3}$ and $10^{-2}(1 \%)$. The effect of boron on $\boldsymbol{\alpha}$ and its temperature dependence is quite marked, shifting $\alpha_{300}$ to a positive peak of $20 \mu \mathrm{V} /{ }^{\circ} \mathrm{K}$ at a B/C ratio of $10^{-3}$. There is a strong correlation of the change in behavior of $\boldsymbol{\alpha}$ and $\mathrm{R}_{0}$ vs boronation at a Fermi level shift of $0.02 \mathrm{eV}$ and a B/C atom ratio of $-2 \times 10^{-4}$, indicating boron's high ionization efficiency. Except at high boron concentrations, these changes are not accompanied by any marked change in the resistivity.
\end{abstract}

\section{INTRODUCTION}

The Fermi surface of a solid, being an energy construct of the charge carrier distribution, can most easily be studied by varying the number of charge carriers it contains. This variation can be obtained by the creation of lattice defects or by the introduction of impurities. However, undue perturbation of the host lattice must be avoided, thus keeping intact the initial energy bands so that the gradual progression from the pure case can be studied. In general, for semimetals, the density of electronic states near the top of the Fermi distribution is low as compared with metals, and thus the addition of any defect that changes the number of carriers can alter the Fermi level. The electronic properties of a small band-overlap material such as graphite become very responsive to this shift. The measurements of greatest interest are those that are sensitive to the difference in electron and hole behavior, or more fundamentally, those that show the effect of the individual

* Based in part on a dissertation by P.S. Grosewald submitted to the Pennsylvania State University in partial fulfillment of the requirements for the degree of Doctor of Philosophy.

+ Present Address: IBM Thomas J. Watson Research Center, Yorktown Heights, New York 10598.
Fermi surfaces without being unduly influenced by scattering effects. The resulting property changes can give information concerning the band structure and also serve to test existing models of that sturcture. In single crystals at low temperatures, the oscillatory galvanomagnetic effects fulfill these requirements, but above $200^{\circ} \mathrm{K}$ and in polycrystalline material the Seebeck or thermoelectric effect most closely satisfies these requirements, since boundary scattering has no first-order effect on the normal thermoelectric or Hall phenomena. The thermoelectric power of a polycrystalline sample is thus equivalent to that of a single crystal, as long as there are no changes in the number of carriers (relative ratios for a multicarrier system, both in number of carriers and their mobility ratios).

It has been known for some time that small amounts of boron have a marked effect on the physical properties of graphite. In 1894 Girard and Street $[1,2]$ patented the use of boric acid to catalyze graphitization. Later, investigations of Weintraub [3], as a sidelight to his work with boron compounds, indicated that the electrical resistance of carbon and the temperature dependence of the resistance could be greatly modified by minute $\left(10^{-4} \mathrm{~B} / \mathrm{C}\right)$ amounts of boron. Until fairly recently, however, no further attempts were made to understand the various processes taking place, beyond speculation that boron either promoted 
graphitization and thus lowered the resistance, or had no effect on crystal growth, but simply added current carriers, or that some combination of both processes prevailed.

Most recent experimental approaches use partially graphitized material and not true graphite. This paper presents the results of a study on the effect of boronation of compacted natural graphite flakes (SP-1) on the electrical resistivity $(\rho)$, the absolute thermoelectric power $(\alpha)$, their temperature dependence, the zero field Hall constant $\left(\mathrm{R}_{\mathrm{O}}\right)$ and the magnetoresistance. Boron was chosen as a relatively ideal acceptor because its ion core size is comparable to that of graphite and because it has one less valence electron than carbon. The use of compacted graphite offers the advantage of large sample size, regular dimensions, and uniform doping over a wide range of boron concentrations. These advantages are not obtainable by any other method of preparation. Soule's single crystal measurements [4-6] of $\rho$ and $\mathrm{R}_{\mathrm{O}}$ allow us to relate polycrystalline properties to single-crystal data. These measurements of $\alpha$ allow us to add thermoelectric data to the list of natural crystal properties being compared to artificial crystal data.

\section{EXPERIMENTAL}

\subsection{Description of Materials Used}

2.1.1 Graphite. The graphite used in this study was from Carbon Products Division, Union Carbide, spectroscopic graphite powder, Catalog No. L4100, grade SP-1, lot F10. The material is a natural (Madagascar flake) graphite specially purified at high temperatures in halogen atmospheres. The total concentration of impurities in this lot was $<1.1$ $\mathrm{ppm}$. The powder is composed of flake-like particles having a median diameter of $30 \mu$ and a modal diameter of $20 \mu$. Taking as a suitable model of the particle a circular slab and assuming that the diameter is much greater than the particle thickness, the average particle thickness is estimated as $0.5 \mu$ from the BET surface area of $1.8 \mathrm{~m}^{2} / \mathrm{g}$ and He density of $2.25 \pm 0.01 \mathrm{~g} / \mathrm{cc}[7]$. Calculations indicate that there are about $10^{18}$ edge atoms $/ \mathrm{cc}$. Individual particles are composed of a small number of highly graphitic crystallites $\{8,9]$.
2.1.2 Boron. The doping material was A.D. Mackay grade AA crystalline boron powder. It has a purity of $>99.5 \%$; its sieve size is -325 mesh or $44 \mu$. The various compounds of boron that might be present (small particles of boron powder are highly reactive with oxygen) are all reduced above $2000^{\circ} \mathrm{C}(10,11)$.

\subsection{Sample Preparation}

The graphite and boron powders were thoroughly mixed to give uniform samples containing approximately $1,10,100,500,1000$, 2000,5000 , and $10,000 \mathrm{ppm}$ boron/carbon (B/C) by weight. Fifteen-gram lots of these samples were pressed for one minute at $10^{5}$ psi to produce solid samples $2-1 / 16 \times 9 / 16 \times$ $9 / 16$ in. This pressure was built up and reduced gradually (about $750 \mathrm{psi} / \mathrm{sec}$.). The long dimension of the pressed samples was perpendicular to the molding direction. Previous studies [12] on the effect of varying the molding pressure and the rate of its application over wide limits revealed a negligible effect on $\alpha$ and $\rho$ when the molding pressure was greater than $5 \times 10^{4}$ psi. Their compacted samples and ours were all milled into rectangular bars $(2 \times 3 / 8 \times 3 / 8$ in. $)$. A hole slightly larger than $1 / 8$ in. was drilled through the center parallel to the long axis.

Samples were then prepared for heat treatment. Bars of the same boron level were placed in a nuclear graphite susceptor and covered with graphite powder, and a nuclear graphite cap was placed over the susceptor. The entire assembly was then lowered into a nuclear graphite crucible which, in turn, was packed with graphite powder (all parts of the furnace having a total impurity content $<1$ ppm). The crucible was part of an induction heater powered by a $30 \mathrm{~kW}$ Ajax-Northrup high-frequency converter. The entire system was flushed with Ar prior to heating and bathed in flowing Ar during the entire heat treatment cycle. The heating cycle consisted of a 40min period to attain a temperature of 2650 $\pm 50^{\circ} \mathrm{C}$, a holding time of $5 \mathrm{~min}$ at this temperature, and about $20 \mathrm{~min}$ to return to $2500^{\circ} \mathrm{C}$. The power was then removed; the unit required 5 hours to cool to room temperature. Based on other studies $\{4,5,13,14]$ the heating cycle above is sufficient to ensure that boron enters uniformly into substitutional sites in the graphite. 


\subsection{Analysis}

Random number tables were used to choose selected heat treated samples for analysis. The samples were sectioned into thirds in their long dimension and then split in half in the other dimension. The random number table was used again to choose various pieces for analysis by chemical, infrared spectroscopy, and mass spectroscopy methods.

Thin sections (1/8 to $1 / 16$ in.) were also cut from two samples parallel and perpendicular to the molding direction, at various depths, for analysis by X-ray reflection diffractometry, as described by Guentert and Klein $[15]$.

\subsection{Instrumentation for Transport Measure- ments}

Since all apparatus used in this study has been completely described elsewhere [16], its features will be summarized very briefly.

The cryostat and its associated measuring equipment were designed to enable an easy interchange of samples and to allow electrical measurements to be carried out in a furnace as well as in a Dewar flask without disturbing the samples and the leads. The cryostat was supported in a Dewar containing a thermal bath: liquid $\mathrm{N}_{2}, \mathrm{~N}_{2}$ vapor above the bath, or air, depending on the temperature range desired. In essence, a specimen was thermally anchored to an evacuated metal chamber, which was then immersed in a liquid or gaseous temperature bath (heat sink). A heater was attached at the free end of the sample to extend the range of temperature attainable. A secondary heater at the fixed end of the sample also provided a means of varying the sample temperature and the temperature gradient in the sample.

Of the available methods of probe contact, the most suitable proved to be that of pressure contacts employing springs. Any other type of pressure contact would either crack the sample or break loose during the dimensional changes following thermal cycling. Platinum probe leads were thermally bonded at the heat sink; all leads (twelve probe, one ground, four heater, two current, and two for the heat sink thermocouple) were brought out of the cryostat through two multiheader seals. Copper-constantan thermocouples, joined to the platinum
文

炭素

probes and referenced with respect to the heat sink, served to determine sink-sample temperature differentials. The heat sink temperature was determined with a copperconstantan premium grade laboratory standard (No.36 wire in double fiber glass sleeving) thermocouple. The heat sink was referenced to an ice bath. Temperature measurements were made by directly recording the thermal electromotive force (emf) generated by the temperature difference between the sample thermocouple junction and the thermocouplecopper lead junction at the heat sink. The emf generated by the temperature difference between the heat sink-thermocouple junction and the ice bath was reduced by a divider network and then was recorded on a multipoint potentiometric recorder (servomechanisms balanced the potentiometer). The potentiometric method insured that the measurement did not disturb the sample steady state, since no current flowed in the circuit at the balance point. It was possible to determine temperatures with a precision of $\pm 0.5 \mathrm{~K}^{\circ}$ and $\pm 0.14 \mathrm{~K}^{\circ}$ at $70^{\circ} \mathrm{K}$ and $500^{\circ} \mathrm{K}$. At these temperatures, $\Delta \mathrm{T}$ could be measured with a precision of $\pm 0.1{ }^{\circ} \mathrm{K}$ and $\pm 0.04^{\circ} \mathrm{K}$, respectively. A $6 \mathrm{~V}$ automobile battery in series with a 0 - 1 mirrored scale milliammeter, a $5 \times 10^{4}$ ohm carbon potentiometer, and the sample provided the current necessary for the determination of the sample resistivity. Drift from a given setting for small currents was not evidenced for periods less than five days.

The potential across the graphite-platinum thermocouple was recorded, first with no current, then with current flowing parallel and then antiparallel to the thermal gradient, and then again with no current. The potentials measured without current flow were used to calculate $\alpha$. The average of the potentials measured with current was also used to calculate $\alpha$, while half the difference was used to calculate $\rho$. The two values of $\alpha$ agreed within the limits of experimental accuracy.

Room temperature Hall and magnetoresistance measurements were made at the Research Laboratory of Alico Speer, Niagrara Falls. 


\section{EXPERIMENTAL RESULTS}

\subsection{Preferred Orientation of Compacted Samples}

Preferred orientation measurements were run on selected samples. In samples doped with $60 \mathrm{ppm}$ of boron, the relative intensity of the $(002)$ reflection $I(\theta)$ as a function of the deviation in degrees from the Bragg angle decreased to a value of 0.5 at $24.5^{\circ}$. For samples with about $1 \%$ boron, $\mathrm{I}(\theta)$ had a value of 0.5 at $31^{\circ}$. All samples showed a tail extending out to high angles.

\subsection{Anisotropy of Electrical Resistivity}

Preliminary measurements of room temperature electrical resistivities perpendicular and parallel to the molding direction and in various orientations perpendicular to the molding direction indicated anisotropy ratios ranging from only 1.5 to 4.0 .

\subsection{Boron Analses}

Table I summarizes the results for the boron content of the samples used. Most analyses were performed by emission spectroscopy and several of the same samples were submitted for chemical analysis. The original SP- 1 graphite contained $<1 \mathrm{ppm}$ boron, as confirmed chemically. As expected, some boron was lost from all samples upon heat treatment, but not a constant percentage. Within a given bar and for bars in the same concentration grouping, analysis agreed within $20 \%$.

\section{Table I}

Boron content of samples

\begin{tabular}{|c|c|c|c|}
\hline \multirow{2}{*}{$\begin{array}{l}\text { SAMPLE } \\
\text { NUMBER }\end{array}$} & \multicolumn{3}{|c|}{ BORON CONCENTRATION, PPM } \\
\hline & $\begin{array}{l}\text { INITIAL } \\
\text { AMOUNT } \\
\text { AFTER } \\
\text { MIXING }\end{array}$ & $\begin{array}{l}\text { EMISSION } \\
\text { SPECTROSCOPY } \\
\text { ANALYSIS }\end{array}$ & $\begin{array}{c}\text { VALUE } \\
\text { USED } \\
\text { (B/C atom ratio) }\end{array}$ \\
\hline $\begin{array}{l}1 \\
9 .\end{array}$ & $\begin{array}{l}\text { Orig. SP-1 } \\
\text { Orig. SP-1 }\end{array}$ & $\begin{array}{l}<1 \Delta \\
<1\end{array}$ & $1 \times 10^{-6}$ \\
\hline $\begin{array}{l}180^{\circ} \\
20\end{array}$ & 11 & $\begin{array}{l}5 \nabla \\
6\end{array}$ & $6 \times 10^{-6}$ \\
\hline $\begin{array}{l}24 \bullet \\
28\end{array}$ & $\begin{array}{l}110 \\
110\end{array}$ & $\begin{array}{l}57 \\
59\end{array}$ & $6 \times 10^{-5}$ \\
\hline $32 \bullet$ & 550 & 140 & $14 \times 10^{-4}$ \\
\hline 39 & 1100 & 1300 & $1 \times 10^{-3}$ \\
\hline $50 \bullet$ & 2200 & 1900 & $2 \times 10^{-3}$ \\
\hline 58 & 5500 & 5000 & $5 \times 10^{-3}$ \\
\hline $63 \cdot$ & 11,100 & 10,600 & $1 \times 10^{-2}$ \\
\hline
\end{tabular}

\subsection{Transport Properties}

3.4.1 Electrical Resistivity. Figure la shows the variation of resistivity with temperature for a group of samples with varying boron content, while Fig. 1 b shows the variation with boron content at $77,150,300$, and $500^{\circ} \mathrm{K}$. The symbol size is a measure of the data scatter except for the undoped SP-1, whose extremely small thermal voltages gave a very low signalto-noise ratio and thus high scatter, as illustrated by the index. Room temperature resistivity for all samples is of the order of $9 \mathrm{x}$ $10^{-4} \mathrm{ohm}-\mathrm{chm}$.

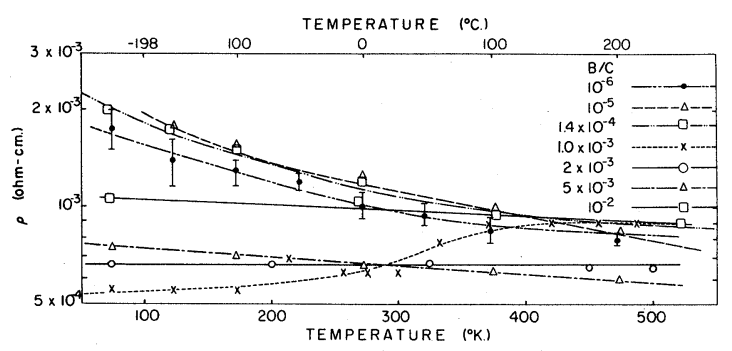

Fig. 1a. Electrical resistivity vs temperature for different boron concentrations.

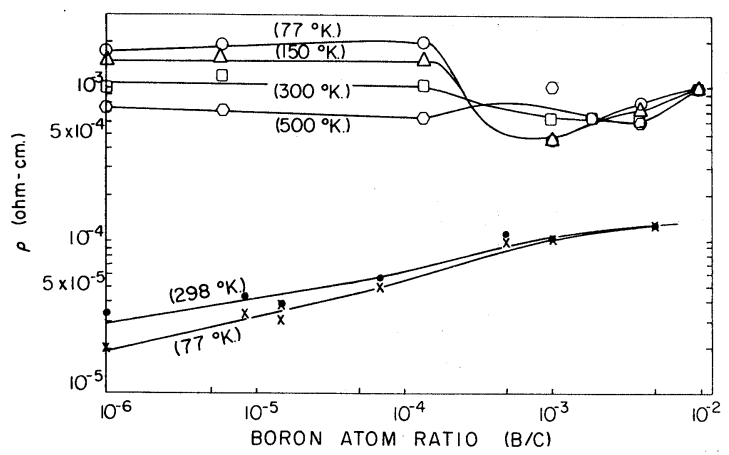

Fig. 1b. Electrical resistivity vs boronation. The lower set of curves indicates the behavior of single-crystal graphite (Ref.6).

\subsubsection{Hall Constant and Magnetoresistance.}

Figures $2 \mathrm{a}$ and $2 \mathrm{~b}$ show the respective ronm temperature $\left(298^{\circ} \mathrm{K}\right)$ variations of $R_{0}$ and $\Delta \rho$ / $\rho_{0}$ at $4.5 \mathrm{kG}$ with boronation. Because of the limited sensitivity of the apparatus and $x-y$ shorting in the samples (current in $\mathrm{x}$-direction, Hall voltage measured in $y$-direction), $\Delta \rho / \rho_{0}$ 
and the small values of $\mathrm{R}_{0}\left(<0.05 \mathrm{~cm}^{3} / \mathrm{coul}\right)$ had to be obtained by statistical analysis of the data (numerical averaging) and, as such, are only correct as order of magnitude values. However, the larger values of $R_{0}\left(>0.05 \mathrm{~cm}^{3}\right.$ / coul) are correct to within the limits of experimental accuracy.

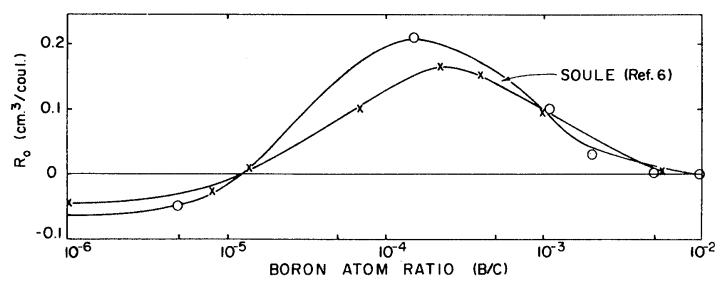

Fig. 2a. Hall effect vs boronation.

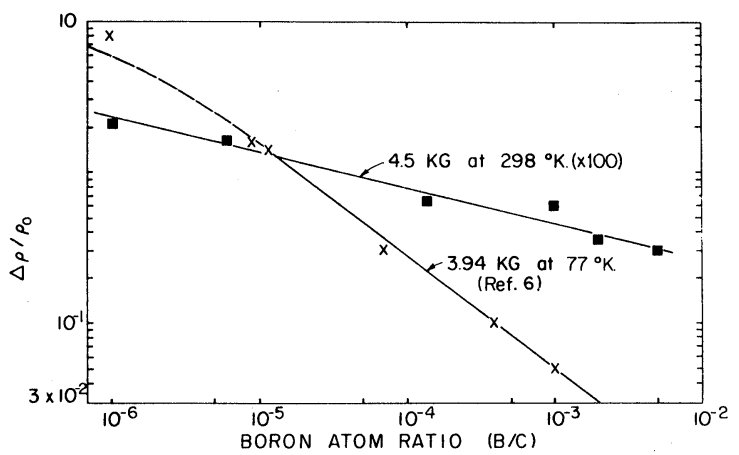

Fig. 2b. Magneto-resistivity vs boronation.

\subsubsection{Absolute Thermoelectric Power.}

Figures 3a and 3b show the variation of $\alpha$ with temperature at fixed boron levels. Figure $3 c$ shows the variation of $\alpha$ with boron content at $77,150,300$, and $500^{\circ} \mathrm{K}$. For the undoped SP- 1 graphite, $\alpha$ decreases with increasing temperature, passing from positive to negative at about $250^{\circ} \mathrm{K}$. The room temperature value is $-1.5 \mu \mathrm{V} /{ }^{\circ} \mathrm{K}$. In contrast to measurements made on carbon samples doped by other methods, no marked scatter of data points was evident.

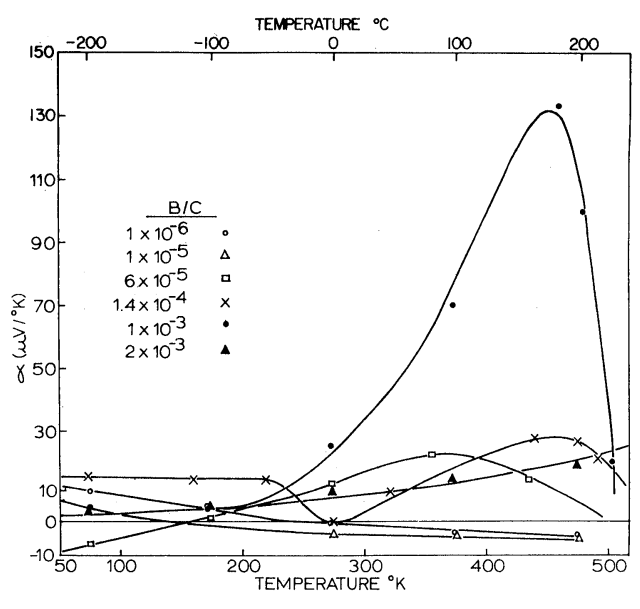

Fig. 3a. Absolute thermoelectric power vs temperature for low boron concentration.

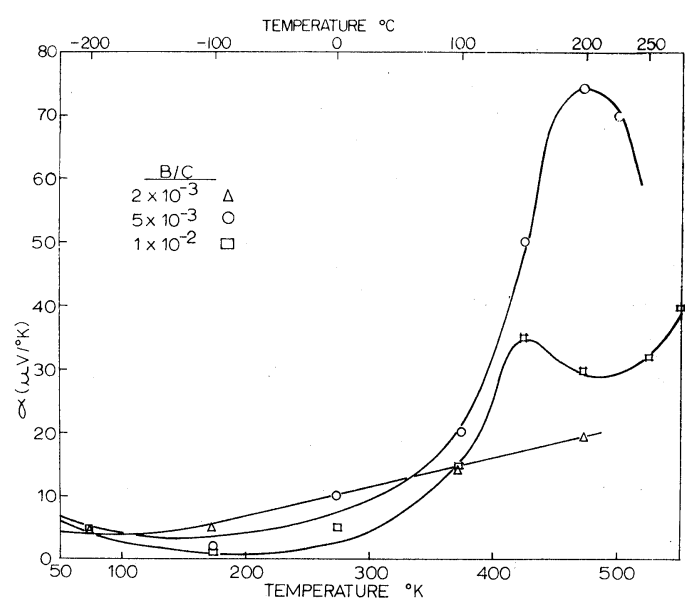

Fig. 3b. Absolute thermoelectric power vs temperature for high (1\%) boron concentrations.

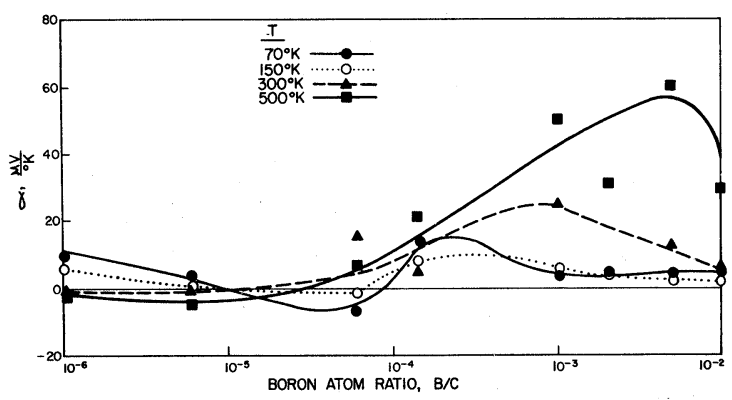

Fig. 3c. Absolute thermoelectric power vs boronation. 


\section{DISCUSSION}

\subsection{Preferred Orientation and Anisotropy}

Price and Bokros [17], using concepts first advanced by Bacon [18] and refined by Ali et al. [19], related prefered orientation curves to thermal expansion and noted that similar techniques would apply to electrical resistivity. Based on this approach, and using a room temperature value of $4.2 \times 10^{-5} \mathrm{ohm}-\mathrm{cm}$ $[4,20]$ for $\rho_{\mathrm{a}}$, the basal plane resistivity, and anisotropy ratios $\left(\sigma_{\mathrm{a}} / \sigma_{\mathrm{c}}\right)$ of 400,1000 and 4000 , calculations of the resistivity perpendicular to the pressing direction yield a value of $4.6 \times 10^{-3} \mathrm{ohm}-\mathrm{cm}$. This value of $\rho_{\mathrm{OX}}$ is essentially independent of $\sigma_{\mathrm{a}} / \sigma_{\mathrm{c}}$, which only has an effect on the third decimal place for the anisotropy ratios chosen.

We may note several points with respect to this result. The effects of intercrystalline resistance and porosity have been neglected in the calculation. Nonetheless, the theoretical resistivity is higher than the experimental value. It can, therefore, be stated that these two factors are of negligible importance in a highly compacted binderless artifact of natural graphite. The differences between the theoretical and experimental resistivities can be accounted for by the approximations used.

Coupling these results with the measured anisotropy ratios eliminates the possibility of using this method of preparation to obtain large mosaic single crystals.

\subsection{Transport Properties and Boronation}

\subsubsection{Absolute thermoelectric power. At} present the lack of a precise theory of $\alpha$ in graphite makes an exact understanding of $\alpha$ vs boronation and temperature difficult to achieve. However, by using an approximate theory, $\alpha$ still is the most interesting property for a study of minute shifts in $\mathrm{n}$ or the Fermi energy $\zeta_{\mathrm{F}}$ since it directly involves the number of carriers, $\mathrm{n}, \zeta_{\mathrm{F}}$, the mobility and the temperature $[7,16,21]$.

The shift of $\alpha_{\max }$ with temperature arises from the thermal broadening of the Fermi distribution; i.e., electrons can be thermally excited into the conduction band at higher temperatures even if $\zeta_{\mathrm{F}}$ has crossed the conduction band edge. The peak and subsequent drop of $\alpha$ vs B/C arises from a change from a balancing between electron and hole effects to a pure hole effect. The peaks in $\alpha$ vs B/C plots occur for higher values of boron than in $\mathrm{R}_{0}$ vs $\mathrm{B} / \mathrm{C}$ plots. This is to be expected, for $\alpha_{\mathrm{e}}$ will increase when $\zeta_{\mathrm{Fe}}$ approaches the band edge [16]. However, there is a marked change in the behavior of $\alpha$ at a $\mathrm{B} / \mathrm{C}$ ratio of approximately $2 \times 10^{-4}$ (Fig. 3c), corresponding to an energy shift of $0.020 \mathrm{eV}$ [16].

The peaks in the $\alpha$ vs $\mathrm{T}$ plots in the twocarrier region also arise from the balancing of the various parameters, particularly the ratios of both the mobilities and the number of carriers. The peaks in the one-carrier region, however, are more difficult to explain without a precise theory of $\alpha$ for graphite. The shift in $\mathrm{d} \alpha / \mathrm{dT}$ for the more heavily doped materials arises from a change from boundary scattering to impurity scattering (roughly a fourfold increase). The peak itself may arise from the balancing of these two effects, but more likely it reflects the incidence of severe perturbations of the band structure: when a boron atom replaces a carbon atom at a B-type site, the A-B overlap potential is changed radically. The removal of degeneracy $\left(T_{D}\right)$ in pure graphite occurs at $\sim 200^{\circ} \mathrm{K}$; the shift from semimetallic to degenerate semiconductor statistics (over 2kTD) would also produce a peak in $\alpha$ vs T. It is quite definite that the peak does not arise from thermally excited electrons. This conclusion is apparent when one considers that $\zeta_{\mathrm{Fh}}$ lies many $\mathrm{kT}$ below the band edge at boron concentrations greater than $0.2 \%$. This situation contrasts sharply with the only other work on $\alpha$ in boronated graphites (other than pyrocarbons), that of Thielke and Shepard [21], who found that $\boldsymbol{\alpha}$ was inversely proportional to $\mathrm{T}$ at low $\mathrm{B} / \mathrm{C}$ atom ratios and remained constant with $\mathrm{T}$ at high boron levels. However, their work was done at high temperatures $\left(1000-3000^{\circ} \mathrm{C}\right)$; and based on Klein's work at $1000^{\circ} \mathrm{C}$ with boronated pyrocarbons $\{22]$ (which are semimetals or metals at such high temperatures), this finding may be possible.

A comparison of the undoped behavior of $\alpha$ vs temperature for our material and for highly graphitic pyrolytic graphite (e.g. C. Klein or A. Ubbelohde) shows an upward shift of $\alpha$ for our materials. This is to be expected since the presence of $10^{18}$ edge sites/cc would trap electrons and cause exactly the shift that does occur. The shift with temper- 
ature from positive to negative values of $\alpha$ arises from the thermally freed electrons. Any other mechanism causing such a shift would also show up in the resistivity. Since it does not, it can be concluded that the trapping sites are shallow traps.

\subsubsection{Electrical Resistivity. At low boron} concentrations the resistivity remains essentially constant with boronation. At these relatively low concentrations the boron is abstracting electrons from the conduction band and adding holes to the valence band, and so the total number of carriers remains constant. This will continue as long as the number of traps is equal to or less than the initial number of free electrons. Combining the constancy of the resistivity with the constancy of the total number of carriers indicates that the mobility and mean free path is also constant.

When the Fermi level drops below the conduction band edge, the carrier concentration should begin to increase. Assuming that the ionization efficiency is $1 / 2$, that there is a linear trapping rate, and that the pure graphite electron Fermi level is $0.020 \mathrm{eV}$ [6] above the bottom of the conduction band, we would expect (based on the theoretically derived equation that the shift in the Fermi level for the electrons can be given by $\Delta \zeta_{\mathrm{Fe}}(\mathrm{eV})=1.98 \mathrm{M}$ $(B / C)$ where $M$ is the ratio of ionized boronations to the total boron content and $\mathrm{B} / \mathrm{C}$ is in atomic percent [16]) a significant shift in the resistivity to occur at a $\mathrm{B} / \mathrm{C}$ atom ratio of about $2 \times 10^{-4}$. This, in fact, is the case, as seen from Fig. 1a. This close experimental and theoretical agreement is a strong indication of a high ionization efficiency for boron in graphite. At higher temperatures this shift in the resistivity (and the band "crossover point") occurs at higher boron levels, since some extra electrons are thermally excited into the conduction band. Further boronation results in an increase in the resistivity, which then becomes temperature-independent.

An examination of the data of Soule [4-6] and Klein [22] relating the number of holes $\left(\mathrm{n}_{h}\right)$ and $\zeta_{\mathrm{f}}$ vs $\mathrm{B} / \mathrm{C}$ also indicates a high ionization efficiency for boron. Empirically, their raw data and their specifically calculated ionization efficiencies lead to multiplicative constants of the order of 2.04 from Soule's work and 3.3 from Klein's data. These values compare favorably with 1.98 , calculated from theory
[16].

Considering the constancy of the mobility with temperature at low boron concentrations, the constancy of the slope of $\rho \mathrm{vs} \mathrm{T}$ with boronation, and the various scattering mechanisms that are temperature-independent, we conclude that boundary scattering predominates, since, if scattering from impurities is not important, the slope of $\rho$ vs B/C should be temperatureindependent [23]. At higher boron content (greater than $0.1 \%$ ) the resistivity increases with boronation and becomes approximately temperature-independent, an indication of ionized impurity scattering dominance.

\subsubsection{Hall Constant and Magnetoresistance.}

These two effects and the conductivity show the results of a shift in the number and types of carriers. A consideration of the general behavior of $R_{0}$ in boronated single crystals shows very good agreement between our results and those of Soule.

The shift between Soule's peak and that found in this study probably is due to the smaller crystallite size and higher defect concentration of our material and the resulting increase in the number of holes initially present.

The product of $\mathrm{R}_{0}$ and $\sigma$ gives the Hall mobility, which is 185,164 , and $60 \mathrm{~cm}^{2} / \mathrm{V}-$ sec. at $\mathrm{B} / \mathrm{C}$ equal to $2 \times 10^{-4}, 10^{-3}$, and $2 \times$ $10^{-3}$, respectively, thus indicating that the mobility is being reduced by ionized impurity scattering.

The corresponding values of $\mathrm{n}_{\mathrm{h}}$ calculated from $R_{O}$ are $3.8 \times 10^{19}, 6.8 \times 10^{19}$ and $1.9 \times$ $10^{20}$ holes $/ \mathrm{cm}^{3}$. These results indicate a high ionization efficiency and are again compatible with Soule's results [4].

If the smaller crystallite size and the temperature differences between our samples and Soule's are taken into account (in our samples leading to smaller mobilities and larger resistivities), the magnetoresistance data in this study appear to be consistent with those reported previously for single crystals [4].

\section{SUMMARY AND CONCLUSIONS}

At present, the thermoelectric power data are too complex to analyze fully without a suitable theory, but the linear temperature- 
dependence is apparent in all samples over a wide temperature range. Rough calculations of the shift in $\zeta_{\mathrm{f}}$ do indicate a high ionization efficiency for boron. The strong correlation between the behavior of the resistivity, Hall constant and $\alpha$ and boron concentration in the doping range $10^{-4}$ to $10^{-3} \mathrm{~B} / \mathrm{C}$ atom ratio is an indication of the validity of this approach.

In all probability, particles of SP-1 are mosaic single crystals. Their defect concentration is fairly low and there are $\sim 10^{18}$ edge sites per $\mathrm{cc}$, but, using the present techniques, it is not possible to mold a powder into the form of a macroscopic single crystal. However, it may be possible that hot-pressing at $\sim 3000^{\circ} \mathrm{C}$ may yield the desired material. In any event, such a compacted material in which the boron is homogeneously distributed offers the best method of studying the properties of macroscopic, polycrystalline graphite as affected by known, controllable amounts of boron.

$\alpha$ has been shown to be very sensitive to minute changes in $\mathrm{n}$ and thus $\zeta_{\mathrm{f}}$. If the behavior at high temperature is as reported by Klein and Thielke et al. , $\alpha$ may be useful for studying the kinetics of the various graphitegas reactions at elevated temperatures, as first reported by Walker and his coworkers [7].

\section{ACKNOWLEDGMENT}

This study was supported by the U.S. Atomic Energy Commission under Contract No.AT(30-1)-1710. One of the authors (P.S.G.) was the holder of an Airco Speer fellowship for a period while this research was in progress. The samples were heattreated at the Airco Speer Research Laboratories. L. Johnson of Battelle-Northwest performed the $\mathrm{x}$-ray orientation studies.

\section{REFERENCES}

1. A.C. Girard and E.A.G. Street, Brev. Franc., 236 (1894).

2. Idem. Bul. Soc. Franc. Electrochem., 12, 268 (1895).
3. E. Weintraub, Trans. Electrochem. Soc., 16, 182 (1909).

4. D.E. Soule, Proc. Third Conference on Carbon, Pergamon Press, New York, 1957, p. 203; idem, private communication.

5. Idem, Phys. Rev., 112, 698 (1958).

6. Idem, Proc. Fifth Conference on Carbon, Pergamon Press, New York, Vol. 1, 1962 , p. 13.

7. P. L. Walker, Jr., L.G. Austin, and J. J. Tietjen, Chemistry and Physics of Carbon, Marcel Dekker, New York, Vol. 1, 1966, p. 328 .

8. J. Lothe, as quoted in J. F. Strange Ph. D. Thesis, The Pennsylvania State University, 1964.

9. S. Irving, private communication, 1964.

10. U.S. Borax Corp., Tech. Data Sheet No. 1A/I, 1960.

11. N. Sidgwick, Chemical Elements and Their Compounds, Vol. 1, Oxford University Press (London), 1959.

12. J.J. Tietjen, Ph. D. Thesis, Pennsylvania State University, 1963.

13. R. V. Sara and C. E. Lowell, Tech. Doc. Report No. WADD TDR-60-143 Part V, 1965.

14. C. E. Lowell, J. Am. Cer. Soc., 50, 142 (1967).

15. O. J. Geuntert and C. A. Klein, Appl. Phys. Letters, 2, 125 (1963).

16. P. S. Grosewald, Ph. D. Thesis, The Pennsylvania State University, 1966.

17. R. J. Price and J. C. Bokros, J. Appl . Phys., 36, 1897 (1965).

18. G. E. Bacon, J. Appl. Chem., 6, 477 (1956).

19. D. Ali, E. Fitzer and A. Ragoss, Industrial Carbon and Graphite Conf. (London), 1958, p. 135.

20. A. W. Smith and N. A. Rasor, Phys. Rev., 104, 892 (1956).

21. N. R. Thielke and R. L. Shepard, USAEC Oak Ridge, High Temperature Thermometry Seminar (USAEC, Washington, 1960), TID-7586 pf 1, p. 44.

22. C. A. Klein, J. Appl. Phys., 35, 2947 (1964).

23. S. Mrozowski, J. Chem. Phys., 21, 492 (1953). 